\title{
The effect of intestinal ischemia on plasma thiol/disulphide homeostasis in an experimental study
}

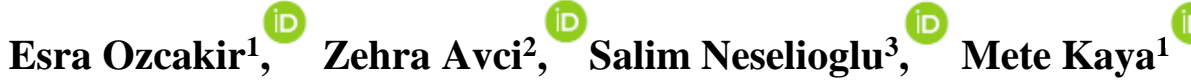 \\ ${ }^{I}$ Department of Pediatric Surgery, University of Health Sciences, Bursa Medical Faculty, Bursa, Turkey \\ ${ }^{2}$ Department of Pathology, Uludag University, Veterinary Faculty, Bursa, Turkey \\ ${ }^{3}$ Department of Biochemistry, Yildirim Beyazit University, Ataturk Training \& Research Hospital, Ankara, Turkey
}

\section{ABSTRACT}

Aim: To investigate the effects of acute intestinal ischemia on plasma thiol/disulphide homeostasis (TDSH), which has been investigated in a limited number of studies in the related literature.

Methods: Twenty-four rats were randomized into control (operation without ischemia, GIS), and ischemia groups (GII-60, GIII-180). For ischemia, the superior mesenteric artery was sutured and the rats were exposed to 60 and 180 minutes of intestinal ischemia, respectively. Plasma TDSH was measured in blood samples collected at the end of the ischemia, and the pathology of ileum segments resected was evaluated.

Results: The experimental ischemic conditions provided were confirmed by the total histopathological scoring system statistically. The levels of serum human albumin and ischemia modified albumin (IMA) in groups were detected in quite a close range of each other. There was no found a statistically significant difference for IMA between groups $(p>0.05)$. The alternations on the levels of plasma TDSH parameters were observed in the study. According to ischemic conditions, the thiol/disulfide ratio fluctuations were detected in the plasma TDSH. The native thiol and total thiol levels seem to have decreased according to ischemia; no statistical difference was detected. In addition, the disulfide levels increasing according to ischemia either was not found significant statistically $(p>0.05)$.

Conclusion: Although this study showed the oxidative balance in intestinal ischemia had affected plasma TDSH, also it revealed that intestinal ischemia didn't create a statistically significant difference between plasma TDSH components.

Key words: Intestinal ischemia, oxidative stress, injury, thiol/disulphide homeostasis, rat.

$\triangle$ Dr. Mete Kaya

Department of Pediatric Surgery, University of Health

Sciences, Bursa Medical Faculty, Bursa, Turkey

E-mail: kayamete@yahoo.com

Received: 2021-06-07 / Revisions: 2021-08-11

Accepted: 2021-08-27 / Published online: 2021-10-01

\section{Introduction}

Intestinal ischemia occurs by reduced intestinal blood flow due to several clinical situations, such as strangulated hernia, intestinal volvulus, necrotizing enterocolitis, small bowel and multi-visceral transplantation, hemorrhagic shock, sepsis, severe burns, trauma, and mesenteric thrombosis/embolism. It results in dysfunction of the gut barrier, which leads to an increase in permeability of the epithelium and infiltration of the intestinal wall by inflammatory cells. These cells cause a systemic inflammatory response by releasing pro-inflammatory cytokines, and finally the delay in diagnosis and treatment may be fatal for the patient is associated with high mortality 
and morbidity [1]. The role of oxidative stress (OS) and reactive oxygen species (ROS) have been shown to contribute to the pathogenesis of intestinal ischemic diseases [2]. The main mechanism causing OS is considered to be lipid peroxidation of mitochondria and cell membranes in ischemic tissue [2,3].

Thiols in the organisms are components that involve the sulfur group and are the essential antioxidant buffers that interact with almost all physiologic oxidants. The majority of the plasma thiol pool consists mainly of human serum albumin (HSA), the other proteins, and the small part consists of thiols with lowmolecular-weight such as cysteine, cysteinyl glycine, glutathione, homocysteine, and cglutamylcysteine [4]. The thiol groups of proteins are transferred to reversible disulphide bond structures by the oxidization of oxidant molecules in the environment. The disulphide bond structures may reduce back to thiol groups, and thus, the thiol-disulphide balance is maintained [4]. In the dynamic plasma thiol/disulphide homeostasis (TDSH) that consists of the native thiol, total thiol, and disulphides, the shift from balance towards disulphides has been shown in various diseases previously [5-7].

The plasma compartment is characterized by having relatively low concentrations of thiols and by the presence of serum albumin as the most abundant one [8]. Ischemia, hypoxia, increased free radicals, and acidosis leads to changes in the molecular structure of serum albumin by decreasing metal binding capacity at $\mathrm{N}$ - terminal. This new isoform of albumin is called ischemic modified albumin (IMA), and its serum levels can be measured [9]. Although studies have recognized IMA as a marker of cardiac ischemia, several investigations of IMA have also shown increased levels in ischemic diseases [10].
In line with the aforementioned theories and literature, the plasma dynamic TDSH of the organism is expected to be affected by intestinal ischemia. However, the studies investigating the effects of intestinal ischemia on plasma TDSH is not found in the related literature. In the present study, we aimed to investigate the effect of intestinal ischemia on the plasma TDSH, and IMA levels, biochemically and histopathologically, and whether it can be used as a predictor of intestinal ischemia.

\section{Materials and methods}

Each experiment was performed in accordance with the National Guidelines for The Use and Care of Laboratory Animals after obtaining the approval of the Animal Experiments Ethics Committee of Uludag University (number: 2017/13/04). Twenty-four Wistar albino rats weighing 300-400 $\mathrm{g}$ were included in the study. All animals were kept on a $12 \mathrm{~h}$ light: $12 \mathrm{~h}$ darkness cycle at $22-24{ }^{\circ} \mathrm{C}$ with free access to food and fasted for $24 \mathrm{~h}$ before the operation, but were allowed free access to water. Rats were divided randomly into three groups, 8 in each: in Group sham (GIS), rat intestines were eviscerated by laparotomy and then put back; the rats in Group II-60 and Group III-180 were exposed to intestinal ischemia for 60 and 180 minutes, respectively. The applied intestinal ischemia model in the current study was described by Megison in 1990 [11]. Briefly, the rats were anesthetized by using 3\% isoflurane; laparotomy was performed by median incision, and the intestines were eviscerated. In ischemic groups, the base of the superior mesenteric artery was identified and ligated by $3 / 0$ silk suture, and intestines were placed back into the abdominal cavity. After 60 minutes of ischemic period for both GIS and GII-60, and 180 minutes for GIII-180, relaparotomy was performed for gathering blood and intestine 
tissue samples without reperfusion. The animals were sacrificed after operation using an intracardiac injection of pentobarbital (45 $\mathrm{mg} / \mathrm{kg}$ ) and open pneumothorax.

\section{Biochemical analysis}

Blood samples were centrifuged to separate the plasma and serum. The serum samples were stored at $-80^{\circ} \mathrm{C}$ until the time of evaluation. Serum TDSH that consists of the native thiol, total thiol, and disulphide was investigated by the novel and automatic spectrophotometric measurement method developed by Erel and Neselioglu; and the disulphide/native thiol ratio, the disulphide/total thiol ratio, and the native thiol/total thiol ratio were calculated [4]. Also, serum IMA levels were measured using the method described by Bar-Or et al. [9]. Serum albumin levels were analyzed using a chemical auto analyzer (Beckman Coulter Chemistry Analyzer AU480, Brea, CA, USA).

\section{Histopathologic examination}

The resected intestinal segments were fixed with formaldehyde (10\%) solution. All samples were embedded in paraffin. Tissues were sectioned in 4-5 $\mu \mathrm{m}$ pieces and stained with hematoxylin-eosin (HE), then analyzed under a light microscope by the same pathologist who was blind to the study. Histopathological findings were graded from 0 to 5 in accordance with the total histopathologic score (THS) defined by Chiu et al. [12].

The grades of the THS are as follows: Grade 0: Normal mucosa, Grade 1: Villous subepithelial detachment formation accompanied by capillary congestion, Grade 2: Subepithelial detachments exerted and a moderate amount of upward push on the mucosa epithelium, Grade 3: Large subepithelial detachments exerted a massive amount of upward push on the mucosa epithelium along the villi, and few denuded villus tips were observed, Grade 4: The villi were denuded to the level of lamina propria and dilated capillaries, Grade 5: Presence of ulceration, the disintegration of lamina propria, and hemorrhage.

\section{Statistical analysis}

Statistical analyses were performed with IBM SPSS ver.23.0 (IBM Corp. Released 2015. IBM SPSS Statistics for Windows, Version 23.0. Armonk, NY: IBM Corp.). The data were examined by the Shapiro Wilk test whether or not it presents normal distribution. The results were presented as mean \pm standard deviation or median (minimum-maximum) for continuous variables including plasma TDSH (native thiol, total thiol, disulphide, disulphide /native thiol ratio, disulphide /total thiol ratio, and native thiol /total thiol ratio) and IMA levels. As the variables were distributed normally, one-way ANOVA was employed to analyze the data. The THS of groups were analyzed as quantitative levels by the Kruskal-Wallis test. The p-value $<0.05$ was considered as statistically significant.

\section{Results}

Histological examination revealed normal small intestines in GIS, while ischemic changes were confirmed in the other experimental groups (Figure 1). The histopathological results are displayed as a boxplot graphic in Figure 1. The THS of ischemia were found significantly higher in the GII-60 and G III-180 groups when compared with GIS ( $p<0.05)$.

The results of total plasma TDSH (native thiol, total thiol, disulphide, disulphide/ total thiol ratio, and native thiol /total thiol ratio), HSA, and IMA levels were summarized in Table 1. The mean HSA and IMA levels in the groups were detected quite close to each other. The mean levels of IMA were displayed in Figure 2A. Although the highest value of IMA within groups was expected in GIII-180, they were observed to course in close range of each other. 

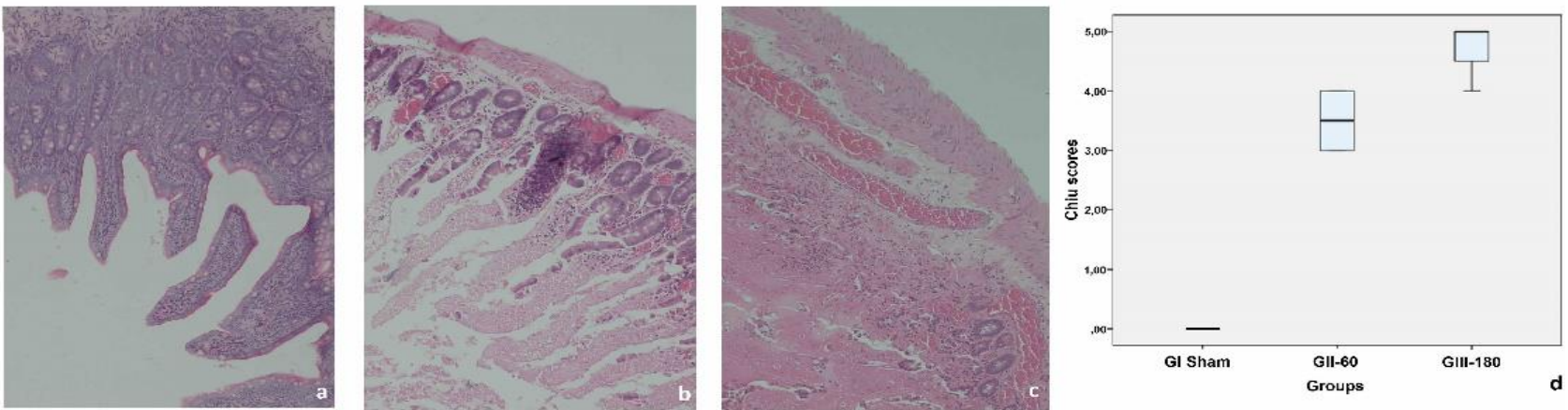

Figure 1. Representative light microscopy (Hematoxylin \& Eosin staining) images of the intestinal epithelium and histopathological scoring. A: Non-ischemic group (GIS) with normal mucosa (Grade 0). B: Ischemia for 60 minutes (GI-60). The denied villi with lamina propria by dilated capillaries (Grade 4). C: Ischemia for 180 minutes (GII-180). The intestinal wall damage with ulceration, the disintegration of lamina propria, and hemorrhage (Grade 5). D: Histopathologic intestinal damage induced by intestinal ischemia was studied using the Chiu score. Each point depicts a sample evaluation ( $\mathrm{n}=8$ per group).

Table 1. The comparison analysis of plasma thiol pool parameters (ischemia-modified albumin, serum albümin, native thiol, total thiol, disulphide, and their ratios).

\begin{tabular}{|c|c|c|c|c|}
\hline Thiol pool parameters & GIS & GII-60 & GIII-180 & $P$ value \\
\hline$H S A(g / d l)$ & $3,49 \pm 0,57$ & $3,298 \pm 0,38$ & $3,423 \pm 0,323$ & 0,684 \\
\hline$I M A(U / m l)$ & $1,289 \pm 0,08$ & $1,300 \pm 0,09$ & $1,209 \pm 0,085$ & 0,097 \\
\hline Native thiol ( $\mu \mathrm{mol} / \mathrm{l})$ & $83,00 \pm 49,67$ & $82,60 \pm 34,10$ & $62,91 \pm 21,23$ & 0,468 \\
\hline Total thiol $(\mu \mathrm{mol} / \mathrm{l})$ & $114,91 \pm 52,45$ & $112,91 \pm 34,60$ & $97,76 \pm 20,69$ & 0,620 \\
\hline Native thiol / total thiol (\%) & $68,19 \pm 10,92$ & $71,50 \pm 12,06$ & $63,38 \pm 11,83$ & 0,392 \\
\hline Disulphide $(\mu \mathrm{mol} / \mathrm{l})$ & $15,957 \pm 2,74$ & $15,156 \pm 5,024$ & $17,425 \pm 4,877$ & 0,589 \\
\hline Disulphide / native thiol (\%) & $24,955 \pm 11,953$ & $21,811 \pm 12,882$ & $31,825 \pm 18,097$ & 0,396 \\
\hline Disulphide / total thiol (\%) & $15,902 \pm 5,463$ & $14,247 \pm 6,031$ & $18,308 \pm 5,916$ & 0,392 \\
\hline
\end{tabular}

Values: Mean \pm SD. HSA: Human serum albumin; IMA: Ischemia-modified albumin; SD: Standart deviation

There was no significant statistical difference between experimental groups for IMA levels (Table 1) $(p>0.05)$.

The native thiol $(62.91 \mu \mathrm{mol} / \mathrm{l})$ and native/total thiol ratio $(63.38 \%)$ were significantly lower in GIII-180 and GII-60 than in GIS (Figure 2B). However, these values were not statistically significantly different between groups $(p=0,468, p=0,392)$ (Table 1). As seen in Figure $2 \mathrm{C}$, the disulphide levels were different among the three groups. Both GIS and GII-60 groups had lower disulphide values compared with the GIII-180 ( $p=15,957$ and $p=15,156$, respectively) (Table 1). Nevertheless, no statistically significant difference in disulphide levels was found between groups. The disulfide/native thiol ratio was significantly higher in the GIII-180 group than in others $(31.82 \%)$. Although the increasing ratio was seen in Figure 2D, there was no statistically 

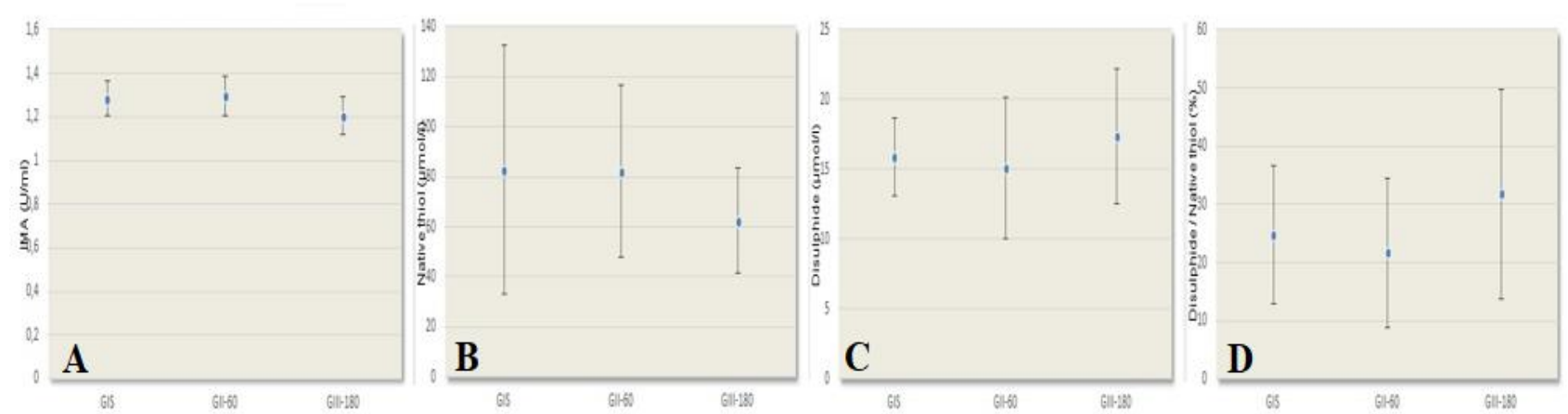

Figure 2. A: The course of serum IMA levels in groups. B: Native thiol levels by groups. GIS had higher native thiol values than both GII-60 and GIII-180. C: Disulphide levels by groups. GIII had higher serum disulphide levels than both groups. D: Disulphide/native thiol ratios by groups. The disulphide/native thiol ratio was higher in the GIII-180 than in others.

significant difference between groups $(p=0,396)($ Table 1).

\section{Discussion}

Intestinal ischemia progresses rapidly and leads to irreversible damage leading to high morbidity and mortality [13]. This severe condition is associated with a progressive inflammatory response causing OS, cellular dysfunction, hemorrhage, and even necrosis [14]. Various biomarkers have recently been reported as a facilitative factor in the process of diagnosing intestinal ischemia, such as acidosis, elevated amylase, D-dimer, intestinal fatty acid-binding protein (I-FABP), serum alpha-glutathione, S-Transferase (alpha-GST), cobalt-albumin binding assay (CABA), procalcitonin, etc. [15]. However, there are no specific laboratory biomarkers in a blood sample for diagnosing intestinal ischemia in daily practice.

IMA is an altered HSA that forms under the conditions of OS and is considered a predictor $[9,14,15]$. In acute ischemic situations, the metal binding capacity of albumin for transition metals such as copper, nickel, and cobalt decreases, resulting in a metabolic variant of the protein commonly known as IMA $[9,10]$. In almost all ischemic or hypoxic clinical studies,
IMA and serum albumin levels were found to be high $[10,16,17]$. Gunduz et al. found that preoperative IMA levels were significantly higher in patients with intestinal ischemia [10]. In the study of Acar et al. they found that IMA levels were high in rats that underwent renal ischemia and reperfusion, although it was not statistically significant [16]. In our study, the IMA levels were not statistically significantly different between ischemic groups. It was considered that the changes of the ischemic tissues may not affect systemic circulation yet, and hence the levels of IMA in ischemic groups are not increased. We also think that the binding affinity of albumin to other metals was not changed since our model produces complete ischemia, not reperfusion.

In 2014, Dr. Erel and Neselioglu developed a novel and automated assay for the organism's antioxidant - oxidant balance, which determined plasma TDSH [4]. Thiols, as a major antioxidant, play an important role in the eradication of ROS via non-enzymatic pathways. The thiol groups of proteins are transferred to reversible disulphide bond structures by the oxidization of oxidant molecules in the environment. In comparison with other parameters, the authors provide an easy, relatively cost-efficient, practical, fully 
automated spectrophotometric assay for the determination of plasma dynamic TDSH. The role of plasma TDSH in various clinical and experimental ischemic studies such as testicular and ovarian torsions, cardiac and renal ischemia, and pulmonary embolism, although most studies are conditions that cause ischemiareperfusion or chronic OS and its importance has been investigated [5,16,18-20]. In the present study, the plasma TDSH was not investigated for ischemia-reperfusion injury. The main purpose was to evaluate whether intestinal ischemia affected plasma levels of thiol and disulphide or not.

The dynamic TDSH has been studied in many different clinical situations and experimental organ ischemia models, and results are inconsistent with each other. Kundi et al. in a study conducted in patients with acute myocardial infarction with chest pain lasting at least 20 minutes, thiol and disulphide levels were found to be significantly lower, but disulphide/thiol levels were found to be higher compared to controls [5]. Similarly to our study, Topuz et al. also found that thiol levels were lower, disulphide levels high, and disulphide/thiol ratios significantly higher in patients who were diagnosed with pulmonary embolism and had high pulmonary embolism severity index compared to controls [18]. Urkmez et al. found that natural, thiol, total thiol and disulphide levels were significantly lower in detorsion after 4 and 8 hours of torsion in the testicular torsion model [19]. They reported that prolongation of ischemia leads to a greater decrease in thiol/ disulphide levels and testicular damage may indicate the prognosis. Avci et al. investigated thiol/disulphide levels in experimental ovarian torsion and found that thiol and disulphide levels increased in the 3hour torsion group, decreased in the detorsion group compared to the sham group, and the ratio of disulphide and thiol groups increased to the thiol groups [20]. Finally, Acar et al. found that the thiol group increased significantly in the reperfusion group in their study in which they performed renal ischemia reperfusion [16]. Considering all previous studies, it is seen that different organs and experimental models have different homeostasis results in ischemic conditions. In the current study, by the literature, as the intestinal ischemia increased; the native thiol and total thiol values decreased and the disulphide and disulphide /native thiol values increased. Although it was shown that while thiol levels were decreased the disulfide values were increased (Figure 3,4,5), no statistically significant difference was found (Table 1).

In addition, we think that the proved intestinal ischemia by the high consistent THS in our study does not reveal a statistically significant difference between TDSH components in plasma. Similarly, Dumlu et al. also conducted a study with hypothesizes that both OS and lipid peroxidation contribute to the pathophysiology of intestinal ischemia [21]. They observed that OS and lipid peroxidation play no significant role in intestinal ischemia and did not provide the expected effects on plasma oxidant and antioxidant capacity accompanied by histopathologic signs of ischemia.

It's well known that ROS, an unstable and cytotoxic reduction product of molecular oxygen, is responsible for producing the increased capillary permeability associated with intestinal ischemia and an increase of OS parameters is expected [22]. In fact, in our study, the thiol/disulphide balance was expected to shift towards disulphides as well, which could be considered as an indicator of OS due to an inflammatory process. Although the shift was observed in the values of the TDSH components in groups per the degree of 
intestinal ischemia, it was anticipated that more considerable differences. Thus, the findings of the current study did not completely result in parallel outcomes with the literature statistically.

We think that these results could have been affected by steady-state concentration due to the plasma kinetic barrier. Because of the plasma kinetic barrier, thiol and disulphides do not stay at equilibrium in plasma. Turell et al. have proposed that the steady-state concentrations are the result of several concurrent actions that are affecting not only total concentrations but also oxidized ratios against reduced thiols [8].

Furthermore, a detailed examination of plasma TDSH by several studies pointed out that plasma concentrations of thiols and oxidized derivatives are affected in such conditions, including the ratios of thiol-disulfide exchanger actions, the rates of thiol oxidation with ROS, and the breakthrough rates of thiol-containing molecules from liver or kidney [23-25]. Hereby, detected thiol and disulphide concentrations in our study are not fully consistent with the expected spectrophotometric measurement for thiol oxidation to disulphide. Within the scope of the aforementioned papers, it is possible that our results might be related to the dynamic process of the plasma thiol pool.

In plasma, total thiols are at a lower concentration than in cells, and the predominant thiol is serum albumin. The serum albumin thiol reacts with a wide variety of oxidant species. These reactions are evidenced by the detection of the thiol oxidized forms of albumin in the circulation that increase in the circumstances related to OS $[8,24,25]$. As it is emphasized by Turell, the nature of oxidant species cannot be elucidated exactly because of the kinetic factors depending on the overlapping of the final products, the concentration of both serum albumin, and potentially competing for targets together with diffusion and compartmentalization aspects [8]. According to these pieces of information, it is considered that the HSA levels of our study groups might have been affected by the mentioned overlapping factor.

As for the limitations of the study, the plasma TDSH is an unstable configuration, and its effects and states in metabolic reactions are unpredictable. Despite the experimentally performed intestinal ischemia in groups, whether or not the results of the parameters of plasma TDSH are real affected indicators or random states may never be known because of the limited number of samples. In addition, it might be stated that as a result of being a relatively novel assay procedure, susceptible to reversible and irreversible modifications. It was not possible to control all of the variables that might potentially affect plasma TDSH. Finally, we were unable to compare the basal plasma thiol/disulphide parameters into our study data because these were not investigated preintervention.

\section{Conclusion}

As a consequence, the mesenteric occlusion caused histopathological and thiol- disulphide balance changes in the current intestinal ischemia model. The parameters of plasma TDSH may have affected by cellular redox signalling in tissue destruction but is insufficient to demonstrate the reflection of acute intestinal ischemia on plasma.

Nevertheless, this is the first study to evaluate the effect of intestinal ischemia on plasma TDSH in a small intestine ischemia model. Therefore, further studies are needed to clarify the interaction between intestinal ischemia and plasma thiol/disulfide pool. 
Acknowledgments: The authors would like to thank Assoc. Professor Guven Ozkaya, for statistical analysis in the Department of Biostatistics at Uludag University, Faculty of Medicine, and also, thanks to Faruk Kucukylldiz, the Veterinary Doctor from the Department of Experimental Animals Research at Uludag University, Faculty of Medicine, for the invaluable help.

Finally, a word of thanks to Raziye Ulker the Labor of Microbiology Department at Uludag University School of Medicine for her technical support.

Funding: The author(s) received no financial support for the research, authorship, and/or publication of this article.

Conflict of Interest: The authors declare that they have no conflict of interest.

\section{Ethical statement:}

The study was approved by Local Clinical Research Ethics Committee (Date and Decision Number: 2017/13/04), and written informed consent was obtained from each subject.

\section{Open Access Statement}

This is an open access journal which means that all content is freely available without charge to the user or his/her institution under the terms of the Creative Commons Attribution NonCommercial License (http://creativecommons.org/licenses/bync/4.0). Users are allowed to read, download, copy, distribute, print, search, or link to the full texts of the articles, without asking prior permission from the publisher or the author.

Copyright (c) 2021: Author (s).

\section{References}

[1]Stringa P, Toledano V, Papa-Gobbi R, et al. Galactomannan as a Potential Modulator of Intestinal Ischemia-Reperfusion Injury. J Surg Res. 2020; 249:232-40.
[2]Thomson A, Hemphill D, Jeejeebhoy KN. OS and antioxidants in intestinal disease. Digestive diseases Dig Dis. 1998;16(3):15258.

[3]Bhattacharyya A, Chattopadhyay R, Mitra S, et al. Oxidative stress: an essential factor in the pathogenesis of gastrointestinal mucosal diseases. Physiol Rev. 2014;94(2):329-54.

[4]Erel O, Neselioglu S. A novel and automated assay for thiol/disulphide homeostasis. Clin Biochem. 2014;47(18):326-32.

[5]Kundi H, Ates I, Kiziltunc E, et al. A novel oxidative stress marker in acute myocardial infarction; thiol/disulphide homeostasis. Am J Emerg Med. 2015; 33(11):1567-71.

[6]Ates I, Kaplan M, Yuksel M, et al. Determination of thiol/disulphide homeostasis in type 1 diabetes mellitus and the factors associated with thiol oxidation. Endocrine. 2016;51(1):47-51.

[7]Gumusyayla S, Vural G, Bektas H, et al. A novel oxidative stress marker in patients with Alzheimer's disease: dynamic thioldisulphide homeostasis. Acta Neuropsychiatr. 2016;28(6):315-20.

[8]Turell L, Radi R, Alvarez B. The thiol pool in human plasma: the central contribution of albumin to redox processes. Free Radic Biol Med. 2013;9(65):244-53.

[9]Bar-Or D, Lau E, Winkler JV. A novel assay for cobalt-albumin binding and its potential as a marker for myocardial ischemia-a preliminary report. J Emerg Med. 2000;19(4):311-15.

[10]Gunduz A, Turedi S, Mentese A, et al. Ischemia-modified albumin in the diagnosis of acute mesenteric ischemia: a preliminary study. Am J Emerg Med. 2008;26(2):202-5.

[11] Megison SM, Horton JW, Chao H, et al. A new model for intestinal ischemia in the rat. J Surg Res. 1990;49(2):168-73. 
[12]Chiu CJ, McArdle AH, Brown R, et al. Intestinal mucosal lesion in low-flow states. Arch Surg. 1970;101(4):478-83.

[13]Kärkkäinen JM, Acosta S Acute mesenteric ischemia (part I) - Incidence, etiologies, and how to improve early diagnosis. Best Pract Res Clin Gastroenterol. 2017;31(1):15-25.

[14]Bala M, Kashuk J, Moore EE, et al. Acute mesenteric ischemia: guidelines of the World Society of Emergency Surgery. World J Emerg Surg. 2017;(7):12-38.

[15] Treskes N, Persoon AM, van Zanten A. Diagnostic accuracy of novel serological biomarkers to detect acute mesenteric ischemia: a systematic review and metaanalysis. Intern Emerg Med. 2017; 12(6):821-36.

[16] Acar M, Kaplan SH, Erdem AF, et al. Effects of dexmedetomidine on new oxidative stress markers on renal ischaemiareperfusion injury in rats: thiol/disulphide homeostasis and the ischaemia-modified albumin. Arch Physiol Biochem. 2020;13;16.

[17]Toker A, Aribas A, Yerlikaya FH, et al. Serum and saliva levels of ischemiamodified albumin in patients with acute myocardial infarction. J Clin Lab Anal. 2013;27(2):99-104.

[18] Topuz M, Kaplan M, Akkus O, et al. The prognostic importance of thiol/disulfide homeostasis in patients with acute pulmonary thromboembolism. Am J Emerg Med. 2016;34(12):2315-19.

[19]Urkmez A, Kutluhan MA, Topaktas R, et al. Prognostic value of thiol/disulphide homeostasis in predicting testicular ischaemia-reperfusion injury in rats. Andrologia. 2018;50(10)e13134.

[20] Ayengin K, Alp HH, Avci V, et al. The effect of laparoscopic and open surgery on oxidative DNA damage and IL-37 in children with acute appendicitis. Ir J Med Sci. 2021;190(1):281-89.

[21]Dumlu EG, Bozkurt B, Tokaç M, et al. Oxidative Stress and Lipid Peroxidation in the Ischemic Small Intestine: Pathological and Biochemical Evaluation in a Rat model of Superior Mesenteric Ischemia. Int Surg. 2014;99(6):868-74.

[22]Parks DA, Granger DN. Ischemia-induced vascular changes: role of xanthine oxidase and hydroxyl radicals. Am J Physiol. 1983;245(2):285-89.

[23] Gallogly MM, Starke DW, Mieyal JJ. Mechanistic and kinetic details of catalysis of thiol-disulfide exchange by glutaredoxins and potential mechanisms of regulation. Antioxid Redox Signal. 2009;11(5):105981.

[24]Turell L, Carballal S, Botti H, et al. Oxidation of the albumin thiol to sulfenic acid and its implications in the intravascular compartment. Braz J Med Biol Res. 2009;42(4):305-11.

[25] Halvey PJ, Watson WH, Hansen JM, et al. Compartmental oxidation of thioldisulphide redox couples during epidermal growth factor signalling. Biochem $\mathrm{J}$. 2005;386(2):215-19. 